\title{
Molecular Architecture of Non-Linear Polymers: Kinetic Modeling and Experimental Characterization of the System Methyl Methacrylate + Ethylene Glycol Dimethacrylate
}

\author{
Ivone M. R. Trigo, ${ }^{1}$ Miguel A. D. Gonçalves, ${ }^{2}$ Rolando C. S. Dias, ${ }^{3}$ \\ Mário Rui P. F. N. Costa ${ }^{* 4}$
}

Summary: A general kinetic approach allowing the prediction of the molecular architecture of non-linear polymers is applied to the study of the copolymerization of methyl methacrylate (MMA) with ethylene glycol dimethacrylate (EGDMA). Dynamic predictions of molecular weight distributions, sequence length distributions and mean square radius of gyration are possible before and after gelation. A set of experiments concerning the copolymerization of MMA and EGDMA was carried out in toluene solution at $60{ }^{\circ} \mathrm{C}$ for which classic radical kinetics is a good approximation. The time evolution of key polymer properties was followed using a SEC system with a refractive index detector coupled with MALLS allowing the determination of absolute weight average molecular weight and apparent molecular size distributions as well as $z$-average radius of gyration. Special focus was given to assess the influence of the initial amount of cross-linker on the dynamics of the non-linear structure build-up of these products. A kinetic scheme comprising 23 different chemical species and 76 chemical reactions was used in the modeling studies of this chemical system. Most of the kinetic parameters used in the simulations have been collected from previous studies. For experiments at low monomer conversion (up to about 0.5) a good agreement between predictions and experimental measurements is observed for molecular weights and z-average radius of gyration by fitting a small number of parameters describing gel effect (with a conversion dependent but chain length independent termination rate parameter) and the relative propagation on pendant double bonds. However, predicted values of weight-average molecular weights and $z$-average radius of gyration before gelation are too low at higher monomer conversions with non-linear systems. The likely cause is the presence of intramolecular reactions which should not be neglected in these circumstances.

Keywords: crosslinking; kinetics; molecular weights; radius of gyration

\section{Introduction}

${ }^{1}$ LSRE-Instituto Politécnico de Bragança, Quinta de S. Apolónia, 5300 Bragança, Portugal Fax: (+351)273313051; E-mail: ivone@ipb.pt

2 LSRE-Instituto Politécnico de Bragança, Quinta de S. Apolónia, 5300 Bragança, Portugal

Fax: (+351)273313051; E-mail: migueldiz@ipb.pt

${ }^{3}$ LSRE-Instituto Politécnico de Bragança, Quinta de S. Apolónia, 5300 Bragança, Portugal Fax: (+351)273313051; E-mail: rdias@ipb.pt

${ }^{4}$ LSRE-Faculdade de Engenharia da Universidade do Porto, Rua Roberto Frias s/n, Porto, 4200-465, Portugal

Fax: (+351)225081666; E-mail: mrcosta@fe.up.pt

The radical copolymerization of monovinyl and multivinyl monomers offers the possibility of synthesizing polymers with a combination of high average molecular weight with a low viscosity; such materials are nowadays often used as rheology modifiers or coatings. Moreover, with these polymerization systems it is also possible to obtain insoluble polymer networks with important applications in a broad range of 
separation processes (such as many derivatives of styrene/divinylbenzene polymers) or as superabsorbents hydrogels. Indeed, materials resulting from vinyl/divinyl copolymerization have found many applications in biomedicine (e.g. contact lenses, smart polymers for drug or gene delivery or dental restorative materials), in tissue engineering, in the production of imprinted polymers, in several important separation processes of pharmaceutical or bioengineering industries or in the production of microelectronic devices. Therefore, it was inevitable the start-up of a growing research activity related with the polymer reaction engineering of vinyl/divinyl copolymerization. Landin and Macosko ${ }^{[1]}$ and Hamielec and coworkers, ${ }^{[2,3]}$ using different modeling approaches with this same chemical system, methyl methacrylate (MMA) + ethylene glycol dimethacrylate (EGDMA), carried out two decades ago two of the most important kinetic studies in this subject and paved the way for the more recent studies.

Nowadays, new research lines have been explored in order to control the synthesis of soluble branched polymers from vinyl and divinyl monomers, namely by trying to attain higher monomer conversions without gelation (e.g. through the use of a chain transfer agent ${ }^{[4,5]}$ or through a semi-batch operation mode $\left.{ }^{[6]}\right)$. Other important experimental works have dealt with the characterization of the molecular architecture of these materials. ${ }^{[4]}$ The application of controlled radical polymerization techniques (CRP) to these polymerization systems is another important research line also currently taking place. Recent works reporting the ATRP copolymerization of methacrylates with divinyl monomers (or the homopolymerization of the latter) can be found in the literature: MMA with EGDMA, ${ }^{[8]}$ homopolymerization of poly(ethylene-glycol)dimethacrylate, ${ }^{[9]}$ 2hydroxypropyl methacrylate with EGDMA or bisphenol-A dimethacrylate ${ }^{[10]}$ homopolymerization of allyl methacrylate, ${ }^{[11,12]}$ 2-(N,N-dimethylamino)ethyl methacrylate with EGDMA, ${ }^{[13]}$ 2-hydroxypropyl metha- crylate with a disulfide based dimethacrylate. ${ }^{[14]}$

In parallel with the experimental investigations carried out on this subject, different kinds of mathematical models ${ }^{[3,15-18]}$ have been developed in order to predict the behavior of these polymerization systems and the structure of the outcoming materials. It is important to note that in these polymerization systems important deviations are often observed with respect to the predictions of Flory-Stockmayer's theory ${ }^{[19,20]}$ or the theory of branching process ${ }^{[21]}$ as well as the mathematically equivalent recursive approach. ${ }^{[22]}$ Intramolecular cyclization and reduced reactivity of pendant double bonds have been recognized as major factors governing the behavior of these polymerization systems. These phenomena also require the introduction of better modeling tools of the statistical build-up of chains, since the link probabilities show considerable changes within the same molecules or the network, invalidating the formerly used simplifying assumptions. Monte Carlo simulation (very slow) or finite element methods have provided a way to circumvent this difficulty.

A general kinetic approach (an extended method of moments) capable of predicting (both before and after gelation) average molecular weights and distributions, sequence length distributions and mean square radius of gyration for non-linear irreversible multicomponent polymerizations has also become a useful tool in this research area. ${ }^{[23-32]}$ The key distinctive features of this approach relative to alternative methods are extensively discussed in the above references. In this work, this general approach is applied to the conventional radical solution polymerization of MMA with EGDMA in a batch reactor. It is shown that besides the prediction of the dynamics of molecular weights it is also possible to obtain the dynamics of the mean square radius of gyration of the polymers. These predictions are compared with measurements by SEC/MALLS for the whole set of different experiments performed. We show that the same fitting 
parameters calculated from the measured molecular weights and monomer conversions yield also good predictions of the mean square radius of gyration whenever intramolecular reactions are negligible. This consolidated knowledge about the molecular architecture of these materials should hopefully be later exploited for the prediction of physical properties relevant for their uses.

\section{Experimental Part}

These experiments were carried out with an automated semi-batch reactor of $2.5 \mathrm{dm}^{3}$ maximum capacity operating in batch mode. A detailed description of this experimental set-up was presented elsewhere. ${ }^{[6]}$

Toluene at $99.7 \%$ purity, AIBN at $98 \%$ purity, benzoyl peroxide (BPO) moistened with $25 \%$ water at $97 \%$ purity (calculated on the dry substance), methyl methacrylate stabilized with 10 to 100 ppm monomethyl ether hydroquinone at $99 \%$ purity and ethylene glycol dimethacrylate stabilized with 100 ppm monomethyl ether hydroquinone at $98 \%$ purity have been purchased from Sigma Aldrich and used as received.

The desired quantities of reactants were charged to the reactor (see Table 1) and brought to the desired temperature $\left(60^{\circ} \mathrm{C}\right)$. Polymerizations were started (defining the instant $t=0$ ) by adding the initiator to the reactor. Argon was bubbled in the reaction vessel at a flow rate $40 \mathrm{~cm}^{3} / \mathrm{min}$ during one hour, before the start of the polymerization in order to deoxygenate the reaction medium, and that same flow rate was kept constant during the whole polymerization process.

Samples of polymer were withdrawn from the reactor at prescribed polymerization times and analyzed in the SEC/ MALLS system as described elsewhere. ${ }^{[6]}$ This integrated system enables the measurements of molecular weights and average molecular radius of gyration. THF at a flowrate of $1 \mathrm{ml} / \mathrm{min}$ was used as the eluent and the SEC system operates at a constant temperature of $30^{\circ} \mathrm{C}$. Refractive index increment $(d n / d c)$ for the polymers, solvent and MMA in THF (respectively 0.0912, 0.1100 and $\left.0.0077 \mathrm{~cm}^{3} / \mathrm{g}\right)$, required for analyzing the MALLS results have also been measured. The overall monomer conversion was measured using SEC information $^{[6]}$ and confirmed by gravimetry.

\section{Kinetic Modeling}

The modeling of the present case study was carried out using the authors' general kinetic approach for describing non-linear irreversible polymerization systems. ${ }^{[23-32]}$ Twenty three different chemical species are considered as shown in Table 2. The copolymerization of MMA with EGDMA, eventually in the presence of an inhibitor and/or a retarder (besides initiator, solvent

Table 1.

Set of experiments performed in the radical copolymerization of MMA with EGDMA in Toluene solution at $60^{\circ} \mathrm{C}$.

\begin{tabular}{lcccccccc}
\hline Exp. & $\begin{array}{c}\text { MMA } \\
\left(\mathrm{dm}^{3}\right)\end{array}$ & $\begin{array}{c}{[\mathrm{MMA}]} \\
\left(\mathrm{mol} / \mathrm{dm}^{3}\right)\end{array}$ & $\begin{array}{c}\text { EGDMA } \\
(\mathrm{g})\end{array}$ & $\begin{array}{c}{[\mathrm{EGDMA}]} \\
\left(\mathrm{mol} / \mathrm{dm}^{3}\right)\end{array}$ & $\mathrm{I}(\mathrm{g})$ & $\begin{array}{c}{[\mathrm{l}]} \\
\left(\mathrm{mol} / \mathrm{dm}^{3}\right)\end{array}$ & $\begin{array}{c}\text { S } \\
\left(\mathrm{dm}^{3}\right)\end{array}$ & $\begin{array}{c}{[\mathrm{S}]} \\
\left(\mathrm{mol}^{3} / \mathrm{dm}^{3}\right)\end{array}$ \\
\hline A & 0.35 & 3.269 & $1.0261(w=0.310 \%)$ & $5.170 \times 10^{-3}$ & $2.5604 \mathrm{AIBN}$ & $1.56 \times 10^{-2}$ & 0.65 & 6.097 \\
B & 0.35 & 3.267 & $1.5270(w=0.460 \%)$ & $7.692 \times 10^{-3}$ & $2.5883 \mathrm{AIBN}$ & $1.57 \times 10^{-2}$ & 0.65 & 6.094 \\
C & 0.35 & 3.272 & 0.0 & 0.0 & $5.0138 \mathrm{BPO}$ & $1.55 \times 10^{-2}$ & 0.65 & 6.103 \\
D & 0.35 & 3.271 & $0.3365(w=0.101 \%)$ & $1.697 \times 10^{-3}$ & $5.0050 \mathrm{BPO}$ & $1.55 \times 10^{-2}$ & 0.65 & 6.101 \\
E & 0.35 & 3.270 & $0.6023(w=0.181 \%)$ & $3.037 \times 10^{-3}$ & $5.0796 \mathrm{BPO}$ & $1.57 \times 10^{-2}$ & 0.65 & 6.099 \\
F & 0.35 & 3.267 & $1.5056(w=0.452 \%)$ & $7.585 \times 10^{-3}$ & $5.0033 \mathrm{BPO}$ & $1.55 \times 10^{-2}$ & 0.65 & 6.094 \\
\hline
\end{tabular}

${ }^{a)}$ Weight fraction of EGDMA defined by $w_{E G D M A}=m_{E G D M A} /\left(m_{E G D M A}+m_{M M A}+m_{l}\right)$.

b) The commercial BPO used in the experiments is moistened with water ( $25 \%$ weight percent of water). The values of the mass of initiator presented in this Table include the water present in BPO. The weight of water was not included in the concentration and weight fraction of BPO. Experiments have been performed with $w_{A I B N}$ around $0.76 \%$ and $w_{B P O}$ around $1.15 \%$. 
Table 2.

Chemical groups for the radical copolymerization of MMA with EGDMA.

\begin{tabular}{lc}
\hline Group Description & Alias \\
\hline Active groups in the polymer & $\mathrm{A}_{1}$ \\
Radical from methyl methacrylate (RMMA) & $\mathrm{A}_{2}$ \\
Radical from ethylene glycol dimethacrylate (REGDMA) & $\mathrm{A}_{3}$ \\
Radical from pendant double bonds (RPDB) & $\mathrm{A}_{4}$ \\
Radical from Retarder (RR) & $\mathrm{M}_{3}$ \\
Pendant double bonds (PDB) & $\mathrm{M}_{1}$ \\
Active groups/reagents not belonging to the polymer & $\mathrm{M}_{2}$ \\
Methyl methacrylate (MMA) & $\mathrm{C}_{1}$ \\
Ethylene glycol dimethacrylate (EGDMA) & $\mathrm{C}_{2}$ \\
Initiator (AlBN or BPO) (I) & $\mathrm{C}_{3}$ \\
Solvent (Toluene) (T) & $\mathrm{C}_{4}$ \\
Chain transfer agent (Carbon-tetrabromide) (CTA) & $\mathrm{C}_{5}$ \\
Inhibitor (Z) & $\mathrm{R}_{1}$ \\
Retarder (R) & $\mathrm{R}_{2}$ \\
Primary radical from initiator (PRI) & $\mathrm{R}_{3}$ \\
Primary radical from solvent (PRT) & $\mathrm{U}_{1}$ \\
Primary radical from chain transfer agent (PRCTA) & $\mathrm{U}_{2}$ \\
Inactive groups in the polymer & $\mathrm{U}_{3}$ \\
Polymerized methyl methacrylate & $\mathrm{F}_{1}$ to $\mathrm{F}_{5}$ \\
Polymerized ethylene glycol dimethacrylate &
\end{tabular}

and a chain transfer agent) is considered. Four different kinds of polymer radicals are distinguished since they present different structures and therefore different reactivities are also plausible. Pendant double bonds arising EGDMA, which are akin of one additional monomer, are also distinguished from the double bonds in the monomers as they are known to show different reactivities. ${ }^{[1-3]}$

In Table 3 the kinetic scheme considered for this polymerization system is presented.

Table 3.

Kinetic scheme considered in the radical copolymerization of MMA with EGDMA.

\begin{tabular}{ll}
\hline Kinetic steps & \multicolumn{1}{c}{ Chemical equation } \\
\hline Initiator decomposition & $C_{1} \stackrel{k_{d}}{\longrightarrow} 2 f R_{1}$ \\
Initiation of monomers and PDBs & $R_{j}+M_{k} \stackrel{k_{l j k}}{\longrightarrow} A_{k}+U_{k}+F_{j}\left(+M_{3}\right.$ if $\left.k=2\right)$ \\
Methyl methacrylate propagation & $A_{i}+M_{1} \stackrel{k_{\text {pil }}}{\longrightarrow} A_{1}+U_{1}$ \\
Ethylene glycol dimethacrylate propagation & $A_{i}+M_{2} \stackrel{k_{\text {piz }}}{\longrightarrow} A_{2}+M_{3}+U_{2}$ \\
PDB propagations & $A_{i}+M_{3} \stackrel{k_{\text {pi3 }}}{\longrightarrow} A_{3}+U_{3}$ \\
Chain transfers to initiator & $A_{i}+C_{1} \stackrel{k_{l i}}{\longrightarrow}$ Dead end $+R_{1}$ \\
Chain transfers to solvent & $A_{i}+C_{2} \stackrel{k_{S i}}{\longrightarrow}$ Dead end $+R_{2}$ \\
Chain transfers to agent & $A_{i}+C_{3} \stackrel{k_{\text {CTAi }}}{\longrightarrow}$ Dead end $+R_{3}$ \\
Chain transfer to MMA & $A_{i}+M_{1} \stackrel{k_{M 1 i}}{\longrightarrow}$ Dead end $+A_{1}+U_{1}$ \\
Chain transfer to EGDMA & $A_{i}+M_{2} \stackrel{k_{M 2 i}}{\longrightarrow}$ Dead end $+A_{2}+M_{3}+U_{2}$ \\
Inhibition of polymer radicals & $A_{i}+C_{4} \stackrel{k_{\text {Zi }}}{\longrightarrow}$ Dead end $+F_{4}$ \\
Inhibition of primary radicals & $R_{i}+C_{4} \stackrel{k_{\text {ZPi }}}{\longrightarrow}$ Inactive Products \\
Retardation of polymer radicals & $A_{i}+C_{5} \stackrel{k_{R i}}{\longrightarrow} A_{5}+F_{5}$ \\
Termination by combination & $A_{i}+A_{j} \stackrel{k_{\text {tcij }}}{\longrightarrow}$ Head - Head Unit \\
Termination by disproportionation & $A_{i}+A_{j} \stackrel{k_{\text {tdij }}}{\longrightarrow}$ Sat. + Unsat. Units \\
\hline
\end{tabular}


A total count of 76 chemical reactions is assumed to exist: initiator decomposition (1), initiation of monomers and PDB by primary radicals (9), propagation of monomers and PDB with the different kinds of polymer radicals (12), chain transfers to solvent (4), chain transfers to CTA (4), inhibition of polymer and primary radicals (7), retardation of carbon centered polymer radicals (3), termination by combination of polymer radicals (10), termination by disproportionation of polymer radicals (10). For the sake of generality, transfers to monomers (12) and to initiator (4) are also considered in this analysis. Despite the somewhat ambiguous distinction between inhibitors (such as monomethyl ether hydroquinone) and retarders (deactivation of primary radicals - deactivation/slowing of polymer radicals), the kinetic steps here considered involving these two species take into account the deactivation of all kinds of radicals and the existence of a polymer radical with a lower reactivity ( $R R$ or $A_{4}$ ) coming from propagation with a retarder (such as oxygen). This radical is supposed to polymerize with monomers, as commonly accepted for oxygen centered radicals polymerizations. ${ }^{[33]}$ Nevertheless, our experimental set-up has avoided this possible issue; simulations have shown that the effect of inhibitor on average molecular weights should only be noticeable above around 500 ppm, which is much higher than what can be expected.

Table 4 and 5 present the numerical values of some of the 77 kinetic parameters used in the simulations. In Table 6 are presented the values considered for the propagation rate coefficients which are the major parameters governing the crosslinking process (see also Figure 1). Past

\section{Table 4.}

Basic set of kinetic parameters considered in the modeling of radical copolymerization of MMA with EGDMA at $60{ }^{\circ} \mathrm{C}$.

\begin{tabular}{|c|c|c|}
\hline Kinetic step & Kinetic parameters involved ${ }^{\text {a) }}$ & Remarks \\
\hline Initiator decomposition & $\begin{array}{l}k_{d}=9.6 \times 10^{-6}\left(\mathrm{~s}^{-1}\right), f_{\mathrm{o}}=0.6(-)(\mathrm{AIBN}) \\
k_{d}=2.8 \times 10^{-6}\left(\mathrm{~s}^{-1}\right), f_{\mathrm{o}}=1(-)(\mathrm{BPO})\end{array}$ & b) c) \\
\hline MMA homopropagation & $k_{p 11}=820$ & d) \\
\hline Polymer radicals termination & $\begin{array}{l}k_{p 11} / \sqrt{k_{t_{0}}}=0.15 \mathrm{dm}^{3 / 2}(\mathrm{~mol} \mathrm{~s})^{-1 / 2} \\
k_{\mathrm{tc}}=\alpha_{\mathrm{tc}} k_{\mathrm{t}} ; k_{\mathrm{td}}=\alpha_{\mathrm{td}} k_{\mathrm{t}} \\
k_{\mathrm{t}}=\left\{\begin{array}{c}k_{\mathrm{to}} \text { if } \quad X<X_{c}=0.3 \\
k_{\mathrm{to}} \exp \left[-Z\left(X-X_{c}\right)\right] \text { if } \quad X>X_{c} ; \quad Z=2\end{array}\right.\end{array}$ & e) \\
\hline Chain transfer to solvent & $C_{S}=k_{S 1} / k_{p 11}=0.2 \times 10^{-4}$ & f) \\
\hline Chain transfer to $\mathrm{CBr}_{4}$ & $C_{C T A}=k_{C T A 1} / k_{p 11}=0.25$ & g) \\
\hline Inhibition & $C_{z}=k_{z 1} / k_{p 11}=10^{4}$ & h) \\
\hline Reaction radical + retarder & $k_{R 1}=10^{9}$ & i) \\
\hline Chain Transfer to Monomer & $C_{M}=k_{M 1} / k_{p 11}=0.2 \times 10^{-4}$ & j) \\
\hline Chain Transfer to Initiator & $C_{l}=k_{11} / k_{p 11}=0.02(\mathrm{BPO}) ;=0(\mathrm{AIBN})$ & k) \\
\hline
\end{tabular}

a) Kinetic parameters units are $\mathrm{dm}^{3} \mathrm{~mol}^{-1} \mathrm{~s}^{-1}$, unless otherwise stated.

b) For AIBN, $k_{d}$ was collected from Ref. [33] (p. 71) and $f_{0}$ from Ref. [35].

c) For BPO, $f k_{d}$ was taken to be the value for a benzene solution measured by inhibition with DPPH ${ }^{[47]}$ which should be more accurate than the previously reported ${ }^{[45]} ; f_{0}$ is known to be above 0.9..$^{[46]}$

d) IUPAC benchmark value ${ }^{[33]}$ collected from Ref. [36].

e) $k_{t}$ is an average termination constant in the framework of the classical kinetics. Scattered values in the range 0.12 to 0.27 for the parameter $k_{p} / \sqrt{k_{t_{0}}}$ can be found in the literature. ${ }^{[37-40]} k_{p} / \sqrt{k_{t_{0}}}=0.15 \mathrm{dm}^{3 / 2}(\mathrm{~mol} \mathrm{~s})^{-1 / 2}$ was estimated from time vs. conversion data (prior to noticeable Norrish-Trommsdorff effect) as measured in the present work. Termination of MMA occurs predominantly by disproportionation $\left(\alpha_{t d}=0.6\right)$ but note that values of $\alpha_{t d}$ from 0.16 to 0.7 are also mentioned in the literature. ${ }^{[33]}$

f) Collected from Ref. [39] (p. 168).

g) Collected from Refs. [2,3].

h) Parameter in the range of the inhibition reactions by some quinones. ${ }^{[33]}$

i) In the range of the rate constant for the reaction of carbon-centered radicals with oxygen. ${ }^{\text {[33] }}$

j) Collected from Ref. [39] (p. 169).

k) Collected from Ref. [40]. 
Table 5.

Assumptions used in the present work for some kinetic parameters considered in the modeling of radical copolymerization of MMA with EGDMA at $60^{\circ} \mathrm{C}$.

\begin{tabular}{llr}
\hline Kinetic step & \multicolumn{1}{c}{ Kinetic parameters involved } & Remarks \\
\hline Initiations & $k_{l k j}=k_{p 1 j} \quad(k=1, \ldots 3, j=1, \ldots, 3)$ & a) \\
Chain transfers to agent and to solvent & $k_{C T A j}=C_{C T A} k_{p j 1}, \quad k_{s j}=C_{s} k_{p j 1}$ & b) \\
Chain transfers to monomers and to initiators & $k_{M i j}=C_{M} k_{p i j}, \quad k_{l j}=C_{l} k_{p j 1}$ & c) \\
Propagations with radicals RR & $k_{p 4 j} / k_{p i j}=0.01 \quad(j=1, \ldots, 3)$ & d) \\
Inhibition & $k_{z i}=C_{z} k_{p i 1} \quad(i=1, \ldots, 4)$ & b) e) \\
Reaction of radicals with retarder & $k_{z P i}=C_{Z} k_{p 11}(i=1, \ldots, 3)$ & b) \\
Termination & $k_{R i} / k_{R 1}=k_{p i 1} / k_{p 11}(i=1, \ldots, 3)$ & f) \\
\hline
\end{tabular}

a) The rate constants for the initiation of the different monomers $(j=1, \ldots, 3)$ with different kinds of primary radicals $(k=1, \ldots 3)$ are considered to take the same values as in the related propagations with RMMA.

b) The rate constants of the reactions between the various propagating radicals with CTA, solvent, inhibitor and retarder are assumed to be proportional to the propagation constant with MMA.

c) The rate constants of transfer to monomers are assumed to be proportional to the similar propagation reactions using the transfer to MMA as reference. The same assumption is used for the reactions of transfer to initiator.

d) The propagation of the monomers with non-carbon centered radicals (e. g. oxygen centered) is considered to be much slower than with the related normal propagation. ${ }^{[33]}$

e) The inhibition of the primary radicals is supposed to occur with MMA at the same extent.

f) It is considered that the kinetic constants for all termination reactions take the same values as the average termination rate constant.

experimental works concerning the copolymerization of MMA with EGDMA have been used for estimating most of the needed kinetic parameters ${ }^{[2,3]}$ involved in this crosslinking polymerization. Nevertheless, here we are distinguishing the radicals arising from PDB (Figure 1) and therefore some other assumptions concerning the reactivity of these species must be used. In the present work a sensitivity analysis with respect to the prediction of the reactivities of these radicals is also presented. Other assumptions are described in the footnotes of the aforementioned Table 4 to 6 .

\section{Results and Discussion}

In Figure 3 are presented the experimentally measured and predicted values of the overall monomer conversion. Those data were used to estimate the parameter $k_{p} / \sqrt{k_{t_{0}}}=0.15 \mathrm{dm}^{3 / 2}(\mathrm{~mol} \mathrm{~s})^{-1 / 2}$ considering valid the classical polymerization kinetics, which was estimated to hold up to monomer conversion $X_{C}=0.3$. A decrease of $k_{t}$ owing to a different regimen of diffusion control was observed for higher monomer conversions and this effect was quantified using an empirical correlation

Table 6.

Propagation rate coefficients $\left(k_{\text {pij }}\right)$ considered in the radical copolymerization MMA with EGDMA at $60^{\circ} \mathrm{C}$.

\begin{tabular}{lcrr}
\hline Radical/Monomer & $\mathbf{M}_{\mathbf{1}}$ & $\mathbf{M}_{\mathbf{2}}$ & $\mathbf{M}_{\mathbf{3}}$ \\
\hline $\mathbf{A}_{\mathbf{1}}$ & $820.0^{\mathrm{a})}$ & $1223.9^{\mathrm{b}}$ & $237.7^{\mathrm{c}}$ \\
$\mathbf{A}_{\mathbf{2}}$ & $1122.7^{\mathrm{b}}$ & $1672.8^{\mathrm{b}}$ & $324.9^{\mathrm{c}}$ \\
$\mathbf{A}_{\mathbf{3}}$ & $598.9^{\mathrm{d}}$ & $895.5^{\mathrm{d}}$ & $173.9^{\mathrm{d}}$ \\
\hline
\end{tabular}

a) IUPAC benchmark value ${ }^{[33]}$ (p. 219).

b) Based on the reactivity ratios $r_{1}=0.67, r_{2}=1.49$ and $k_{p 22} / k_{\text {p11 }}=2.04$ collected from Refs. [2,3].

c) Based on the reactivity ratio $r_{13}=k_{p 11} / k_{p 13}=3.45$ obtained in this work from experimental data and on the ratios $k_{p 13} / k_{p 23}=k_{p 12} / k_{p 22}$ as suggested in Refs. [2,3].

d) It is considered in this work that a geometric decay relation holds for the reactivity of radicals $A_{3}$ with the different kind of monomers: $k_{p 3 j}=\left(k_{p 1 j}\right)^{2} / k_{p 2 j}$. These reactivity decreases are plausible owing to steric factors. The effect of this assumption is also discussed in the current work. 


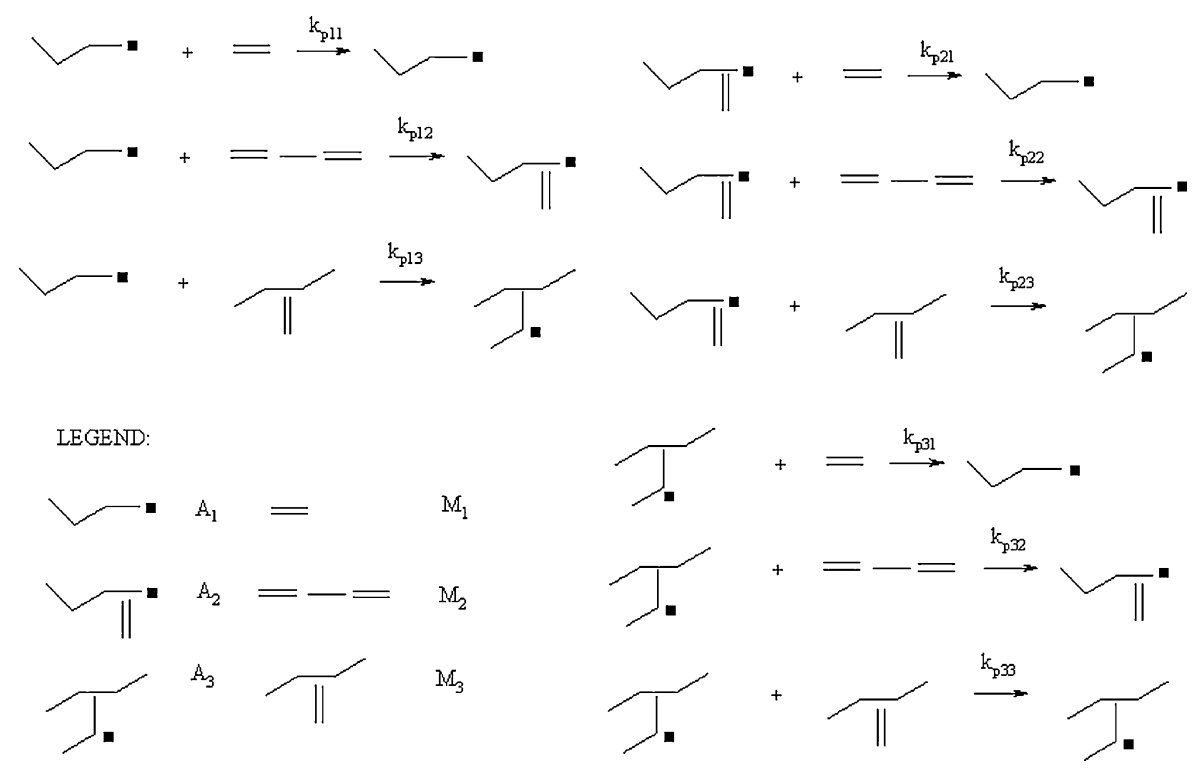

Figure 1.

Schematic representation of the propagation reactions considered in the radical copolymerization MMA with EGDMA (for the sake of simplicity propagations with radicals RR are not here presented).

for the decrease of the termination rate constant as proposed by Tobita and Hamielec $^{[34]}$ (see Table 4).

These solution data do not show the slight influence of the presence of EGDMA on $k_{t}$ which is nevertheless known to occur with bulk polymerization when $w_{E G D M A}$ is increased from 0 to $0.01 .^{[3]}$

In Figure 4(a) and 4(b) are compared the experimental measurements and theoretical predictions for $\bar{M}_{w}$ in the non-linear polymerization MMA + EGDMA initiated by AIBN and BPO. Error bars presented in those two figures for the experimental measurements of this work are those directly indicated by the software of the SEC/RI/MALLS system. The experimental data on $\bar{M}_{w}$ lead to an estimation of the reactivity ratio of $\mathrm{PDB}$, which is $r_{13}=$ $k_{p 11} / k_{p 13}=3.45$. With this apparent reactivity ratio, good agreement between measurements and predictions for runs carried out with different amounts of EGDMA was achieved except when the reaction conditions are favorable to the occurrence of intramolecular reactions (high monomer conversion with non-linear systems). It is important to note that this reactivity ratio is consistent with a decrease of reactivity of $\operatorname{PDB}\left(C_{P}=2 k_{p 13} / k_{p 12}=0.39\right)$, as reported in other works on this subject. ${ }^{[1-3]}$ Further studies in order to clarify the values of PDB reactivities (which actually should be higher than these apparent values) should include experiments at higher dilutions in order to assess the intramolecular cyclization effects. ${ }^{[1,27]}$

Note that the kinetic approach we use lumps all isomers with same counts of groups into the same conventional chemical species. Therefore, an accurate consideration of intramolecular reactions is possible only for the smallest sized loops. ${ }^{[27]}$ It is nevertheless conceivable that models using empirical pseudo-rate constants of cyclization might prove to be useful. They present some mathematical difficulties in their implementation when generating functions are introduced and this has delayed their development by the authors.

The most innovative results of this work are presented in Figures 5(a) and 5(b), where experimental $z$-average mean-square molecular radius of gyration $\left(\bar{R}_{g}\right)$ of the 


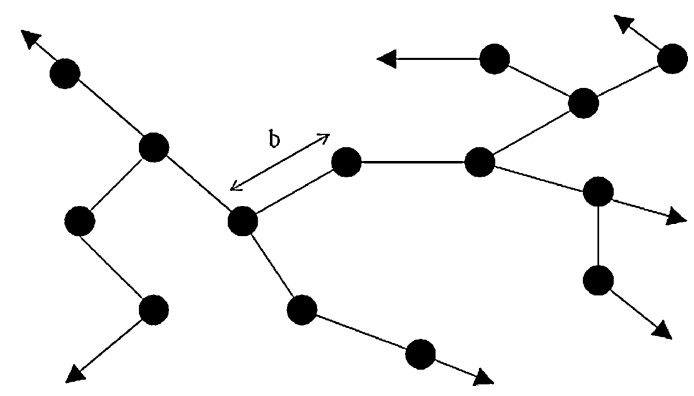

Figure 2.

Schematic representation of the concept of bond length used in the calculation of the average radius of gyration of branched/crosslinked polymers.

synthesized copolymers are compared with the predictions of the present kinetic approach. ${ }^{[31]}$ This method is valid for tree-like polymers with Gaussian chains considered as a set of beads connected by massless freely rotating rods (see Figure 2).

Our predictions are therefore valid at $\Theta$ conditions. Molecular expansion in good solvents (the excluded volume effect) should be taken into account because the experimental measurements were performed in THF at $30^{\circ} \mathrm{C}$. The length of the Kuhn segment connecting centers of mass of repeating units $b=0.615 \mathrm{~nm}$ was estimated using the relation $\bar{R}_{g, l i n_{\theta}}=$ $0.0251 M^{0.5}$ valid for poly(methyl methacrylate) in a $\Theta$ solvent. ${ }^{[42,43]}$ Using the published value for poly(methyl methacrylate) in THF, $\bar{R}_{g, l i n}=0.011 M^{0.596}$ at

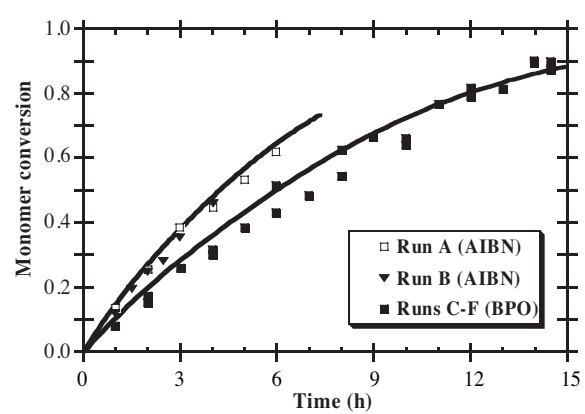

Figure 3.

Time evolution of the measured and predicted overall monomer conversion for different polymerization runs (see Table 1) performed with AIBN and BPO as initiators.
$30{ }^{\circ} \mathrm{C}^{[43]}$ we estimate that also in THF at $30^{\circ} \mathrm{C}$ for linear polydispersed poly(methyl methacrylate) $\bar{R}_{g, l i n} / \bar{R}_{g, l i n_{\theta}}=0.438 M_{z}^{0.096}$.

An extension of our method for taking into account the presence of the excluded volume effect has not yet been developed $^{[31]}$ and in this work we have assumed equality of the expansion factors for

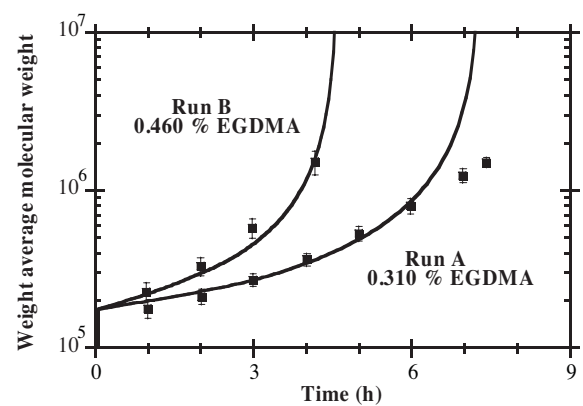

(a)

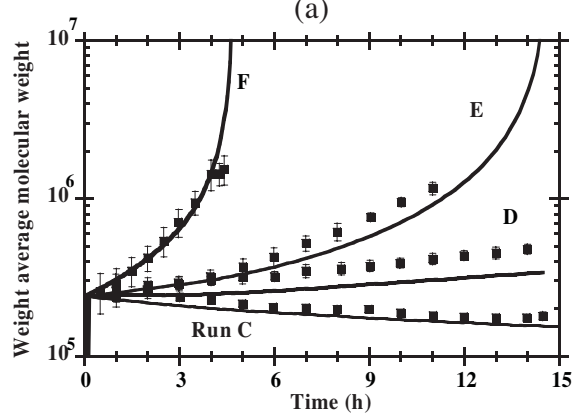

(b)

Figure 4.

(a) Time evolution of the measured and predicted $\bar{M}_{w}$ for polymerization runs performed with AIBN. (b) Same comparison for runs initiated by BPO. 
branched and linear polymer molecules. Several other researchers ${ }^{[48]}$ have also considered this assumption in more recent works with similar goals as this one, but a more exact estimation of $g$ is urgently needed.

In spite of these assumptions, a good agreement is often observed between the predictions and the experimental measurements of $\bar{R}_{g}$ when intramolecular reactions are less important and therefore some reliable information concerning the molecular architecture of these polymers can be obtained using this approach. The exceptions are, as it could be expected, the high discrepancies between predictions and measurements with runs $\mathrm{A}$ and $\mathrm{E}$ where higher monomer conversions at gel point are observed.

The complexity of kinetic schemes such as the one here discussed precludes the direct use of other approaches (such as Monte Carlo simulation or molecular dynamics) which should be improved in order to efficiently describe real linear or branched chains. ${ }^{[49]}$

A major advantage of this general kinetic approach is the possibility of considering more complex kinetic schemes whenever desired. In the present work, radicals from pendant double bonds $\left(\mathrm{A}_{3}\right)$ were distinguished from radicals of MMA or EGDMA because it is plausible that they present different reactivities (see also Table 6). In Figure 6(a) is presented the predicted effect of the reactivity of RPDB on the time evolution of $\bar{M}_{w}$, using run B as an example. Different values of the reactivities of RPDB $k_{p 3 j}^{*}=C_{R} k_{p 3 j}$ were considered in the simulations using the values $\left(k_{p 3 j}\right)$ presented in Table 6 as reference. It can be observed that these parameters can have a major effect on the predictions only when these radicals are reacting much more slowly $\left(C_{R}<0.01\right)$ than the reference situation we have considered. However, this is a plausible situation due to the lower mobility of these radicals and therefore the estimated apparent reactivity ratios of PDB can also be affected by this phenomenon.

The reliability of the predictions of the present kinetic approach was also assessed using experimental data previously obtained for the bulk polymerization of MMA/EGDMA. ${ }^{[2,3]}$ Considering the polymerization conditions and the kinetic parameters used in these works, our predictions of the monomer conversion at gel point were compared with the experimental values observed in the presence of $\mathrm{CBr}_{4}$ as CTA. This comparison is presented in Figure 6(b) and the good agreement between the predictions and measurements confirms the correctness of the foundations of this method.

In Figure 7 is presented the relation between measured average radius of gyration and average molecular weight for linear MMA and different non-linear samples of MMA/EGDMA synthesized a)

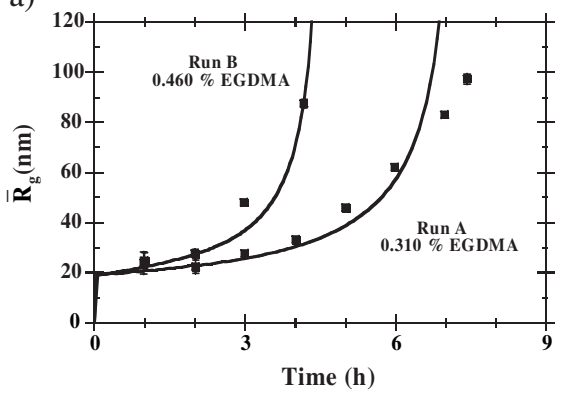

b)

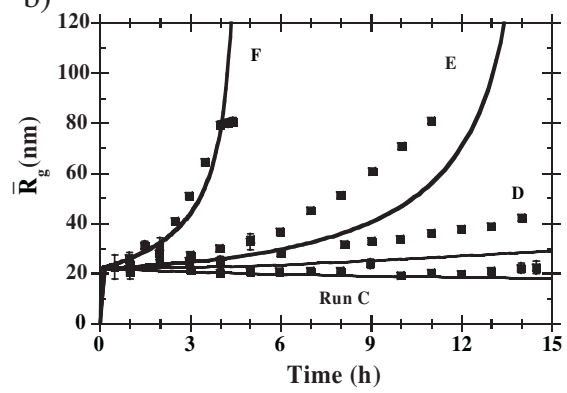

Figure 5 .

(a) Predicted and measured time evolution of $\bar{R}_{g}(\mathrm{~nm})$ in the copolymerization system MMA/EGDMA initiated by AIBN. (b) Same comparison for runs with BPO as initiator. 


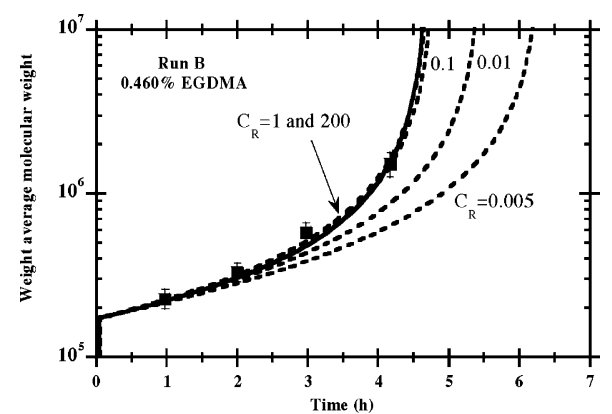

(a)

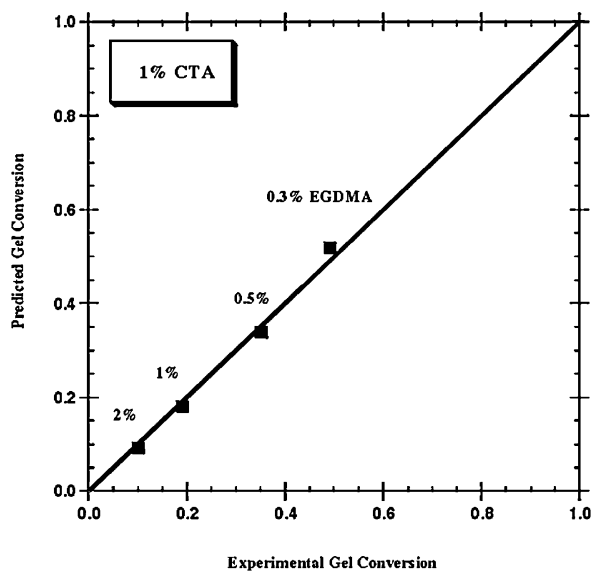

(b)

Figure 6.

(a) The predicted effect of the reactivity of radicals of pendant double bonds on the time evolution of $\bar{M}_{w}$. (b) Predicted and measured gel conversion for the system MMA/EGDMA in the presence of CTA. Experimental data collected from Li, Hamielec and Crowe $^{[2,3]}$ and predictions of the present kinetic model.

in this work. The well known decrease of the size of non-linear polymers when compared with the linear analogous is here confirmed. Nevertheless, it is important to recall that these polymer samples are highly dispersed in molecular mass and the correct comparison is made using $\bar{M}_{z}$ as a reference instead of $\bar{M}_{w}$. Indeed, for a population of linear polymer molecules a power relation (with exponent 0.5 for Gaussian chains) between $\bar{R}_{g}$ (z-average radius of gyration) and $\bar{M}_{z}$ should be observed (see for instance Ref. [32]). Note in the same Figure 7 the high estimated errors in the

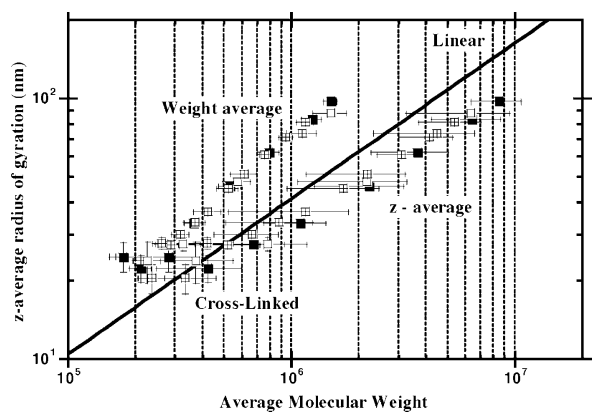

Figure 7.

Relation between average radius of gyration and average molecular weight for linear MMA and several non-linear samples of MMA/EGDMA synthesized in this work.

measurements of $\bar{M}_{z}$ by SEC/MALLS (only $\bar{M}_{w}$ can be directly measured) when highly dispersed polymer samples are analyzed, as previously presented for the system styrene + divinylbenzene. ${ }^{[6]}$

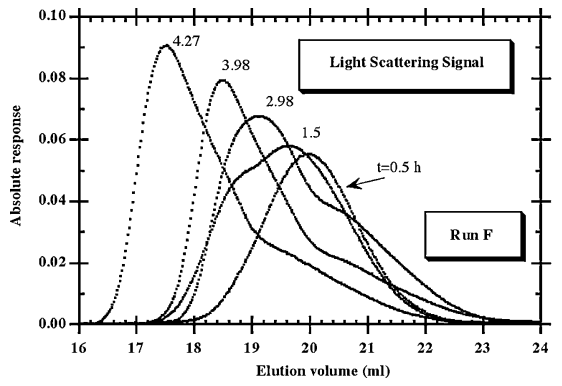

(a)

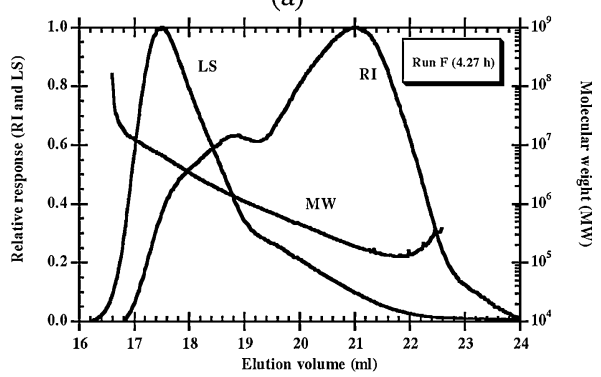

(b)

\section{Figure 8.}

(a) Observed $90^{\circ}$ light scattering signal in the SEC chromatograms of samples of MMA/EGDMA corresponding to different polymerization times. (b) Molecular weight along the SEC chromatogram for a sample of MMA/EGDMA. 


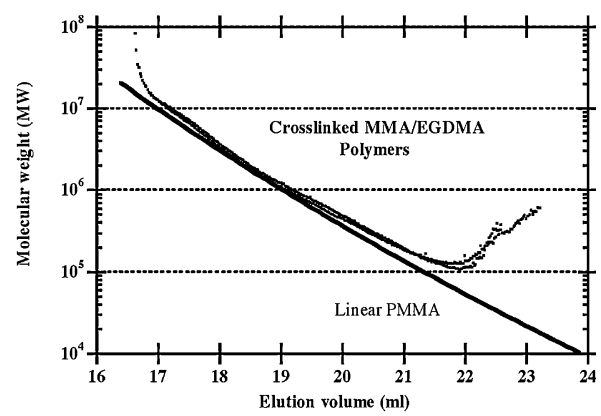

(a)

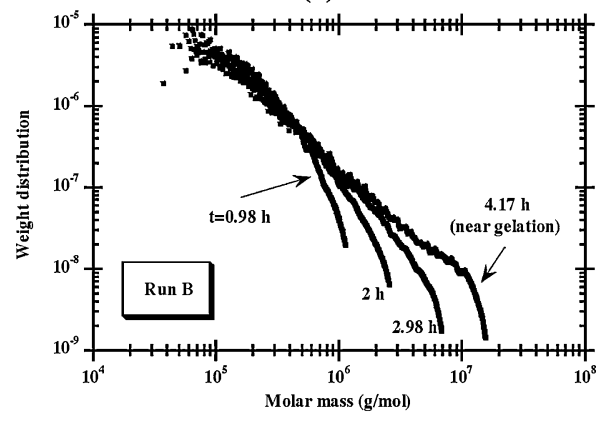

(b)

Figure 9.

(a) Observed relations molecular weight vs. elution volume for MMA/EGDMA copolymers. (b) Time evolution of the molecular weight distribution of MMA/ EGDMA copolymerization showing the formation of a long end tail as gelation is approached.

In Figure 8(a) is presented the dynamics of formation of a polymer population with large dimensions but at a low concentration (forming a kind of cluster) as the gel point is approached. The chromatogram presented in Figure 8(b) of a MMA/EGDMA sample collected close to gel point shows in detail a molecular fraction with a strong light scattering signal but a weak refractive index.

Figure 9(a) shows an inversion in the relation molecular mass vs. elution volume of two highly cross-linked samples of MMA/EGDMA confirming again the existence in the samples of polymer species with same molecular weight but very different molecular sizes. Similar observations have also been recently reported in the literature. ${ }^{[10]}$ Figure 9 (b) presents the time evolution of the chromatograms of MMA/EGDMA samples showing the formation of a long end tail as the gel point is approached, in agreement with previous theoretical results. ${ }^{[28]}$

\section{Conclusions}

In this work, a general kinetic approach was applied to the study of the copolymerization of MMA with EGDMA in toluene solution at $60^{\circ} \mathrm{C}$ and using batch operation mode. A complex kinetic scheme comprising 23 different chemical species and 76 chemical reactions was applied in the modeling studies of this chemical system considering the absence of cyclization intramolecular reactions. Most of the used kinetic parameters have been collected from related studies and experimental measurements by SEC/RI/MALLS of the average molecular weights and monomer conversion of the synthesized products were used to estimate fitting parameters concerning the reaction rate and the reactivity of pendant double bonds. The decrease of the reactivity of pendant double bonds measured in this work is consistent with earlier studies with the same chemical system. ${ }^{[1-3]}$

For experiments at low monomer conversion (up to around 0.5) a good agreement between predictions and experimental measurements is observed. Predicted weight average molecular weights and $z$-average radius of gyration are too low at higher monomer conversions with nonlinear systems. Further experimental studies with more emphasis on describing cyclization effects should be undertaken in order to clarify these issues.

Using the same set of kinetic parameters applied in the predictions of average molecular weights, we show that the measured $z$-average radius of gyration can also be calculated.

It has also been confirmed that the interpretation of the SEC chromatograms of branched/crosslinked polymers may be troublesome due to the existence of 
copolymer chains with the same molecular weight but quite different molecular sizes (elution volumes).

The results here presented are a step towards further studies allowing a better control on the molecular architecture of non-linear polymers with several important applications in biotechnology and medicine (e.g. as "smart" polymers ${ }^{[50]}$ ). The operation in semi-batch reactor ${ }^{[6,51]}$ with controlled radical polymerization (CRP), namely atom transfer radical polymer (ATRP), is a promising combination to improve the properties of these materials. The simplified description valid at low conversion with negligible cyclizations is likely to hold in most cases with CRP as shown in previous works. ${ }^{[52]}$

Acknowledgements: Financial support by Fundação para a Ciência e a Tecnologia (FCT), Ministry of Science and Technology of Portugal and European Community through FEDER (projects $\mathrm{POCI} / \mathrm{EQU} / 44784 / 2002$ and $\mathrm{POCI} /$ EQU/60483/2004) is gratefully acknowledged.

[1] D. T. Landin, C. W. Macosko, Macromolecules 1988, 21, 846.

[2] W.-H. Li, A. E. Hamielec, C. M. Crowe, Polymer 1989, 30, 1513.

[3] W.-H. Li, A. E. Hamielec, C. M. Crowe, Polymer 1989, 30, 1518.

[4] N. O'Brien, A. McKee, D. C. Sherrington, A. T. Slark, A. Titterton, Polymer 2000, 41, 6027.

[5] S. P. Gretton-Watson, E. Alpay, J. H. G. Steinke, J. S. Higgins, Chem. Eng. Sci. 2006, 61, 1421.

[6] M. A. D. Gonçalves, R. C. S. Dias, M. R. P. F. N. Costa, Macromol. Symp. 2007, 259, 124.

[7] G. Saunders, P. A. G. Cormack, S. Graham, D. C. Sherrington, Macromolecules 2005, 38, 6418.

[8] A. R. Wang, S. Zhu, Polym. Engng. Sci. 2005, 45, 720. [9] O. Yu, F. Zeng, S. Zhu, Macromolecules 2001, 34, 1612.

[10] I. Bannister, N. C. Billingham, S. P. Armes, S. P. Rannard, P. Findlay, Macromolecules 2006, 39, 7483. [11] R. Nagelsdiek, M. Mennicken, B. Maier, H. Keul, H. Höcker, Macromolecules 2004, 37, 8923.

[12] R. París, J. L. De La Fuente, J. Polym. Sci. Polym. Chem. Ed. 2005, 43, 2395.

[13] C. Jiang, Y. Shen, S. Zhu, D. Hunkeler, J. Polym. Sci. Polym. Chem. Ed. 2001, 39, 3780.

[14] Y. Li, S. P. Armes, Macromolecules 2005, 38, 8155.
[15] S. Zhu, A. E. Hamielec, Macromolecules 1992, 25, 5457.

[16] S. Zhu, A. E. Hamielec, Macromolecules 1993, 26, 3131.

[17] S. Zhu, Macromolecules 1996, 29, 456.

[18] S. Kizilel, G. Papavasiliou, J. Gossage, F. Teymour, Macromol. React. Eng. 2007, 1, 587.

[19] P. J. Flory, J. Am. Chem. Soc. 1941, 63, 3096.

[20] W. H. Stockmayer, J. Chem. Phys. 1944, 12, 125.

[21] M. Gordon, Proc. R. Soc. London 1962, 268, 240.

[22] C. W. Macosko, D. R. Miller, Macromolecules 1976, 9, 199.

[23] M. R. P. F. N. Costa, R. C. S. Dias, Chem. Eng. Sci. 1994, 49, 421.

[24] M. R. P. F. N. Costa, R. C. S. Dias, Macromol. Theory Simul. 2003, 12, 560.

[25] R. C. S. Dias, M. R. P. F. N. Costa, Macromolecules 2003, 36, 8853.

[26] M. R. P. F. N. Costa, R. C. S. Dias, Chem. Eng. Sci. 2005, 60, 423 .

[27] R. C. S. Dias, M. R. P. F. N. Costa, Polymer 2005, 46, 6163.

[28] R. C. S. Dias, M. R. P. F. N. Costa, Macromol. Theory Simul. 2005, 14, 243.

[29] R. C. S. Dias, M. R. P. F. N. Costa, Polymer 2006, 47, 6895.

[30] M. R. P. F. N. Costa, R. C. S. Dias, Macromol. Symp. 2006, 243, 72.

[31] M. R. P. F. N. Costa, R. C. S. Dias, Polymer 2007, 48, 1785.

[32] R. C. S. Dias, M. R. P. F. N. Costa, Macromol. React. Eng. 2007, 1, 440.

[33] G. Moad, D. H. Solomon, "The Chemistry of Radical Polymerization", $2^{\text {nd }}$ Ed., Elsevier, Oxford 2006.

[34] H. Tobita, A. E. Hamielec, Macromolecules 1989, 22, 3098.

[35] J. C. Bevington, Trans. Faraday Soc. 1955, 51, 1392.

[36] S. Beuermann, M. Buback, T. P. Davis, R. G. Gilbert, R. A. Hutchinson, O. F. Olaj, G. T. Russell, J. Schweer, A. M. van Herk, Makromol. Chem. Phys. 1997, 198, 1545 .

[37] M. S. Matheson, E. E. Auer, E. B. Bevilacqua, E. J. Hart, J Am. Chem. Soc. 1949, 71, 497.

[38] M. Fernández-Garcia, J. J. Martinez, E. L. Madruga, Polymer 1998, 39, 991.

[39] R. A. Hutchinson, Handbook of Polymer Reaction Engineering, Wiley- $\mathrm{VCH}, 2005$.

[40] J. Brandrup, E. H. Immergut, E. A. Grulke, A. Abe, D. R. Bloch, "Polymer Handbook", $4^{\text {th }}$ Ed., Wiley, N. York 1999.

[41] S. Beuermann, M. Buback, Prog. Polym. Sci. 2002, 27, 191.

[42] S. Ioan, M. Bercea, C. Ioan, B. C. Simionescu, Eur. Polym. J. 1995, 31, 85.

[43] J. Búrdalo, R. Medrano, E. Saiz, M. P. Tarazona, Polymer 2000, 41, 1615. 
[44] C. Jackson, Y.-J. Chen, J. W. Mays, J. Appl. Polym. Sci. 1996, 59, 179.

[45] C. A. Barson, J. C. Bevington, J. Polymer Sci. A: Polym. Chem. Ed. 1997, 35, 2955.

[46] G. Moad, D. H. Solomon, S. R. Johns, R. J. Willing, Macromolecules 1982, 15, 1188.

[47] J. C. Bevington, B. J. Hunt, J. Warburton, Polymer 2003, 44, 3469.

[48] Z. Dobkowski, J. Appl. Polym. Sci. 1985, 30, 355 .
[49] M. O. Steinhauser, J. Chem. Phys. 2005, 122, 094901.

[50] I. Galaev, B. Mattiasson, "Smart Polymers Applications in Biotechnology and Biomedicine", $2^{\text {nd }}$ Ed., CRC Press, 2008.

[51] M. A. D. Gonçalves, R. C. S. Dias, M. R. P. F. N. Costa, 2007 AIChE Annual Meeting, Salt Lake City, Utah, 2007.

[52] H. Gao, K. Min, K. Matyjaszewski, Macromolecules 2007, 40, 7763 . 